\title{
AN ALGORITHM OF BOUNDARIES DETECTION IN LOW-CONTRAST RADAR IMAGES OF THE EARTH
}

\author{
Leonid Dorosinskiy ${ }^{1}$, Tamara Lysenko ${ }^{2}$ \\ ${ }^{1,2}$ Ural Federal University \\ 620002, Mir Street 19, Ekaterinburg, RUSSIAN FEDERATION
}

\begin{abstract}
One of the problems of radar images analysis of the Earth is the detection of boarders between areas with different normalized effective radar cross-sections. In this paper, we propose a computationally effective quasi-optimal algorithm capable for building approximation of such boarders with straight line segments for low-contrast radar images and arbitrary line for high-contrast radar images. To achieve computational efficiency we apply image segmentation and later approximation. Efficiency of the proposed algorithm was examined on a number of computer generated radar image fragments including lowcontrast radar images. Proposed algorithm can be effectively implemented using modern parallel computation systems.
\end{abstract}

AMS Subject Classification: 60G35, 93E10, 94A12

Key Words: radar imaging, image recognition, boarder detection, effective radar crosssection

\section{Introduction}

In the Earth radar imaging, observed objects are represented by areas with different normalized effective radar cross-sections. Obtained radar images can contain more or less distinct boundaries between areas of different contrast. The problem of automatic radar images processing contains recognition and

Received: $\quad$ August 27, 2016

Revised: $\quad$ October 4, 2016

Published: November 9, 2016

$\S$ Correspondence author (c) 2016 Academic Publications, Ltd. url: www.acadpubl.eu 
estimation of properties of such boundaries. Along with synthesis of contour recognition algorithms, their analysis is also of large importance. Parameters to be evaluated are: the minimal difference in normalized effective echoing areas, which enables boundaries recognition in low-contrast radar images; influence of a priori uncertainty of surface properties on precision of boundaries recognition [1]. Synthesis of computation-efficient quasi-optimal algorithms and research of their relation to the optimal algorithms also has a big practical value. Our research is aimed at solutions of these problems.

\section{Optimal Solution}

An examined radar image is represented by a two-dimensional array of distinct intensity samples (the intensity is the square of the absolute value) of the observed signal. The probability density of these samples has exponential distribution with mathematical expectation proportional to normalized effective radar cross-section, which corresponds to the Gaussian distribution of an input signal.

If a radar image contains $M$ areas of different contrast $\Omega_{1}, \ldots, \Omega_{M}$, each sample of observed signal $u_{i j}$ has the probability density $W_{k}\left(u_{i j}\right)$ corresponding to the $\Omega_{k}$-th area of the image. An optimal in terms of maximum likelihood boundary detection algorithm would be to calculate

$$
W\left(U / \Omega_{1}, \ldots, \Omega_{M}\right)=\prod_{k=1, i, j \in \Omega_{k}}^{M} \prod W_{k}\left(u_{i j}\right)
$$

for all possible boarders between $M$ areas and to select a configuration having the maximum value of eqn (1). Straightforward implementation of this algorithm is impractical due to its enormous computation complexity. Essential simplification can be achieved by breaking an image into relatively small fragments and approximation of contour lines by a function $y=f(x)$ from the selected family, e.g. polynomial ones. Although this approach narrows the valid boundaries class, the total number of variants to examine is too high; so, the choice of the optimal contour selection is still practically impossible.

\section{Boarder Approximation}

A big number of the Earth radar images have straight-line or relatively smooth boundaries between areas of different radiocontrast. Such contours are typical for radar images of agricultural fields, forestation areas, and a range of 
other artificial and natural objects. Smooth lines separate sea surface areas of different speed and direction of the near-surface wind. Coastlines [2], ice covering borders [3], and sea surface areas polluted by oil have a similar type of boundaries.

In such cases the contour lines within small image fragments can be effectively approximated by straight lines. A fragment size should be chosen according to a priory information about smoothness of the contour line. Thus, in such cases, the problem of boundaries detection can be reduced to detection of straight boundaries between two areas of different radiocontrast within each image fragment and subsequent merging them into larger contour lines [4].

Let a fragment of a radar image has dimensions $A \times D$ and consists of two areas of different contrast $\Omega_{0}$ and $\Omega_{1}$, separated by a straight line $y=k x+b$.

Logarithm of the likelihood function of a vector $U$ can be represented as:

$$
\ln W\left(U / k, b, \sigma_{0}, \sigma_{1}\right)=\left(\sigma_{0} / \sigma_{1}-1\right) \sigma_{0}^{-1} \sum_{i=1}^{D} \sum_{i=1}^{c_{j}} u_{i j}-\ln \left(\sigma_{0} / \sigma_{1}\right) \sum_{j=1}^{D} c_{j}
$$

Here $\sigma_{0}, \sigma_{1}$ are the mean values of normalized effective radar cross-sections for $\Omega_{0}$ and $\Omega_{1}$;

$\left.c_{j}=\right](j-b) / k[$ - azimuthal leap coordinate is the boundary point of the $j$-th distance channel;

With unknown $\sigma_{0}$ and $\sigma_{1}$, the likelihood function of the vector $U$ is

$$
\begin{aligned}
& W(U / k)=\prod_{j=1}^{D}\left\{\prod_{i=1}^{c_{j}}\left[c_{j}\left(\sum_{k=1}^{c_{i}} u_{k j}\right)^{-1} \exp \left\{c_{j}\left(\sum_{k=1}^{c_{j}} u_{k j}\right)^{-1} u_{i j}\right\}\right] \times\right. \\
& \prod_{k=c_{j}+1}^{A}\left[\left(A-c_{j}\right)\left(\sum_{k=c_{j}+1}^{A} u_{k j}\right)^{-1} \exp \left\{-\left(A-c_{j}\right)\left(\sum_{k=c_{j}+1}^{A} u_{k j} u^{-1} u_{i j}\right\}\right],\right.
\end{aligned}
$$

where $\sigma_{0}, \sigma_{1}$ are (optimal in terms of maximum of likelihood) estimations of the mean values of the signal power corresponding to areas $\Omega_{0}$ and $\Omega_{1}$. Taking logarithm of eqn (3), we get:

$$
\begin{aligned}
\ln W\left(\frac{U}{k}\right)= & \sum_{j=1}^{D}\left\{-c_{j} \ln \left(-c_{j}^{-1} \sum_{k=1}^{c_{j}} u_{k j}\right)-\right. \\
& \left.-\left(A-c_{j}\right) \ln \left[\left(A-c_{j}\right)^{-1} \sum_{k=c_{j}+1}^{A} u_{k j}\right]\right\} .
\end{aligned}
$$




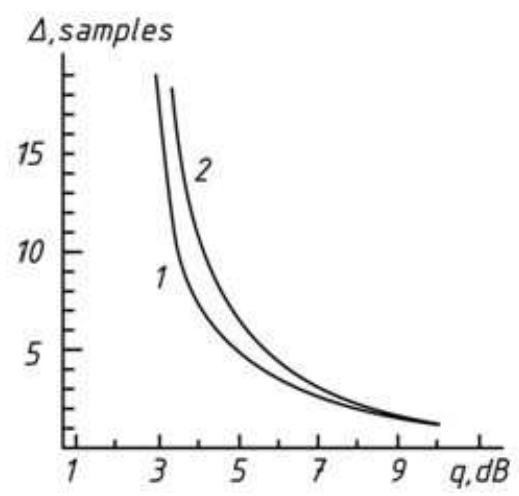

Figure 1: Error of estimation versus radar image contrast

Parameters of a straight boarder are found by maximizing values of eqn (2) or eqn (4). Although these calculations are relatively simple, the solution is still computation expensive for the big number of image fragments. To overcome this difficulty we propose a quasi-optimal algorithm of boundaries detection.

\section{Optimized Algorithm}

The algorithm has two stages. At the first stage, progressive (by distance or azimuth channels) processing is performed to reveal the coordinates of boarder points represented by leaps of the mean value of the instantaneous value of the power of a reflected signal. The processing is implemented according to eqn (2) or eqn (4), but for one-dimensional case. At the second stage, an approximation of obtained set of points with a straight line is performed using the method of cluster analysis. This method provides better accuracy (comparing to the least square errors and the eigenvector methods) in reproduction of boundaries, especially, in cases of small difference in normalized effective radar cross-sections. It is since our method does not take into account explicitly abnormal points.

Analysis of the proposed algorithm was performed using digital statistical models of radar images represented by two-dimensional discrete arrays of random variables with the given probability densities. Using real radar images for conducting a statistical experiment was problematic due to absence of sufficient number of different images of the same surface fragment with accurate coordinate links taken in the same or in close conditions.

Results of the algorithm's first stage analysis are represented by the graph of the mean error of obtained leap coordinate on the contrast $q=\sigma_{0} / \sigma_{1}$ between 


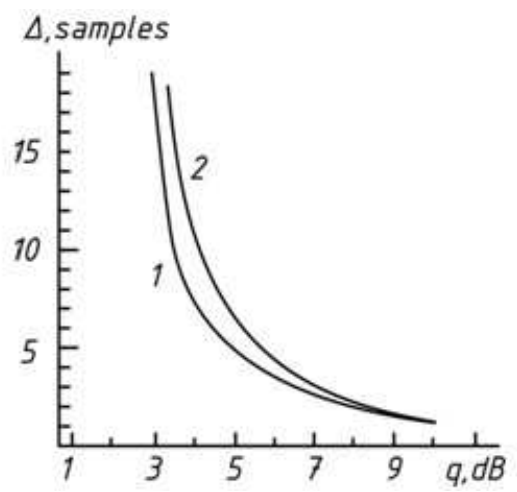

Figure 2: Model of a radar image fragment

areas being separated (Figure 1). Plot 1 is built for the case of known $\sigma_{0}$ and $\sigma_{1}$, plot 2 is obtained for unknown $\sigma_{0}$ and $\sigma_{1}$. The mean error was calculated as the sum of the offset and standard deviation of leap coordinate estimation. Averaging was performed on 30 radar images of size $50 \times 100$ elements. Experiment showed that performance of the algorithm which uses estimations of $\sigma_{0}$ and $\sigma_{1}$, is very close to its performance for a priory known $\sigma_{0}$ and $\sigma_{1}$. If the ratio $\sigma_{0} / \sigma_{1}$, is greater than $8 \mathrm{~dB}$, the coordinate measuring error is about $1-2$ resolution elements, which is almost enough to build an arbitrary separating line basing solely on the results of the first stage of the algorithm.

Figure 2. shows $100 \times 100$ radar image with two areas $\Omega_{0}$ and $\Omega_{1}$ and a solid separating line connecting estimated leaps points. Parameters set for this model were $\sigma_{0}=2, \sigma_{1}=20$, and recovered estimations were $\hat{\sigma}_{0}=2.02$, $\hat{\sigma}_{1}=20.08$, which corresponds to the error less than $2 \%$.

In the case of smaller contrast $(4<q<8)$, errors of estimations of leap coordinates are much higher; so, building the separating line is impossible at the first stage. If a separating line is close to the straight line within an image fragment, it can be recovered using cluster analysis at the second stage of the algorithm. Accuracy of straight boarder reproduction was calculated as the mean of error

$$
\Delta=\sqrt{\left(\theta_{0}-\theta^{\prime}\right)^{2}+\left(\rho_{0}-\rho^{\prime}\right)^{2}} .
$$

Here $\theta^{\prime}$ and $\rho^{\prime}$ are the parameters of the boarder used in modelling; $\theta_{0}$ and $\rho_{0}$ are the parameters recovered by the algorithm.

Figure 3 shows plots of absolute error values eqn (5) versus radar image contrast. Plot 1 is built for the optimal algorithm eqn (2), plot 2 was obtained for the quasi-optimal algorithm with known $\sigma_{0}$ and $\sigma_{1}$, and plot 2' corresponds 


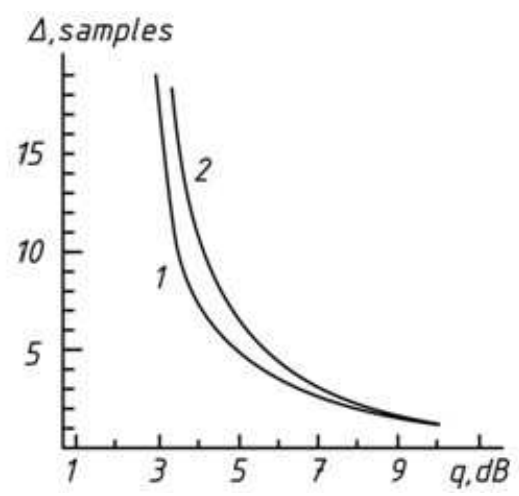

Figure 3: Error of boarder reconstruction versus radar image contrast

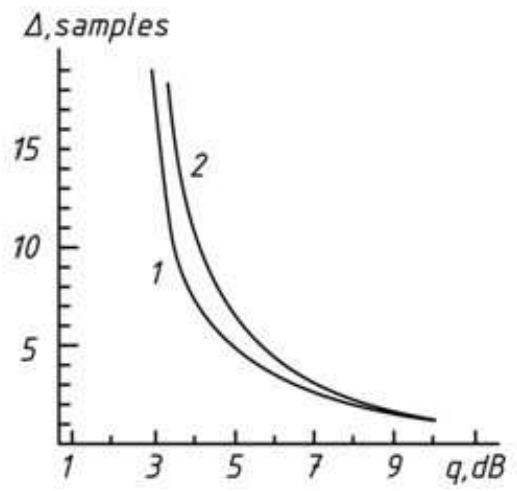

Figure 4: Choosing separating line parameters from multiple options

to the quasi-optimal algorithm with unknown $\sigma_{0}$ and $\sigma_{1}$.

If $q>4 d B$, the quasi-optimal algorithm performs in pair with the optimal algorithm. It is much easier to implement and does not require any a priory knowledge of the different normalized effective radar cross-sections of areas being separated.

For smaller contrast values $(q<3 d B)$, errors of leaps coordinates estimations increase significantly. This causes greater dispersion of leap points within the fragment plane and sometimes results in finding multiple possible separating lines with the cluster analysis method. In this case, we modify the cluster analysis method in the following way.

On the plane $\theta \times \rho$ of straight line parameters points $\left\{\theta_{i}, \rho_{i}\right\}$ are plotted for each built line. Mean coordinates $\left\{\theta_{0}, \rho_{0}\right\}$ are calculated for the largest cluster using methods like those described in [4]. Obtained parameters $\theta, \rho_{0}$ are 


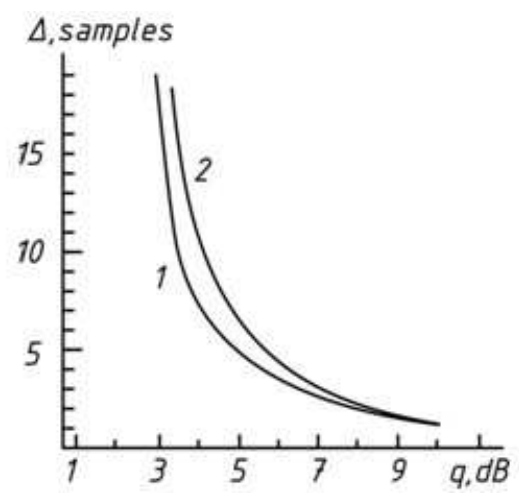

Figure 5: Straight boarders built for a low-contrast radar image fragment

considered to be the parameters of the resulting single separating line.

Figure 5 shows straight boarders between areas $\Omega_{0}$ and $\Omega_{1}$ built using described method for ten low-contrast images generated for the same area fragment with different contrast values $q$ between $0.5 \mathrm{~dB}$ and $2 \mathrm{~dB}$. The line in dashes is the original separating one.

\section{Conclusion}

We have proposed and researched an algorithm of boundaries detection in radar images of the Earth. Proposed algorithm is capable for detecting relatively smooth boundaries, which can be approximated with straight lines within small image fragments for radar image contrast between $3 \mathrm{~dB}$ and $8 \mathrm{~dB}$, and arbitrary boarders for greater contrast values. We have also proposed a modification of the algorithm for radar image contrast less than $3 \mathrm{~dB}$.

\section{References}

[1] L.G. Dorosinsky, Radar signals class recognition algorithm synthesis, In: 24-th International Crimean Conference "Microwave \&5 Telecommunication Technology" (CriMiCo'2014) Conference Proceedings, September 7-13, 2014, Sevastopol: Weber Publishing, 2 (2014), 1137-1138.

[2] L.G. Dorosinsky. The research of the distributed objects' radar image recognition algorithms, In: 23-rd International Crimean Conference "Microwave 85 Telecommunication Technology" (CriMiCo'2013) Conference Proceedings, September 8-13, 2013, Sevastopol: Weber Publishing, 2 (2013), 1216-1217. 
[3] R.O. Duda, P.E. Hart. Pattern Classification and Scene Analysis, Wiley-Interscience, Oxford, 1973.

[4] A.M. Chandra, S.K. Ghosh, Remote sensing and geographical information system, Narosa Pub. House, New Delhi, 2006. 